\title{
METODO INTEGRADO PARA LA GESTION DE UNIVERSIDADES BASADO EN EL BALANCED SCORECARD (BSC) Y EL MODELO EUROPEO DE CALIDAD (EFQM): CASO U.C.S.M.
}

\author{
EDWING JESÚS TICSE VILLANUEVA \\ HORACIO VICENTE BARREDA TAMAYO
}

\begin{abstract}
Resumen
El presente Trabajo realiza la propuesta de un Método Integrado que permite mejorar la competitividad en la Gestión de las Universidades, este método se basa:

- En una herramienta de Gestión Estratégica: el Balanced Scorecard (BSC), que fue creada por Kaplan y Norton en 1992, y

- En el Modelo Europeo de Calidad (EFQM), que fue desarrollado como un Modelo de Excelencia en 1991

La metodología utilizada consiste en analizar las características, ventajas y limitaciones del BSC y el EFQM para aplicarlos simultáneamente en la gestión de Tomando como base los 9 criterios del EFQM, se desarrolla un Mapa Estratégico del $\mathrm{BSC}$, para que se alineen todas las perspectivas hacia el logro de los Objetivos Estratégicos de la Organización

El método integrado planteado se aplica en el caso de la Universidad Católica de Santa María (Arequipa- Perú), donde se analiza las ventajas que implicaría la implementación del mismo
\end{abstract}

Palabras Clave:

EFQM (European Foundation for Quality Management), BSC (Balanced Scorecard), Método Integrado de Gestión Universitaria.

\section{INTRODUCCION}

La medición del desempeño organizacional es un punto central, en tal sentido, Kaplan y Norton (1992) desarrollaron una herramienta innovadora -conocida como Balanced Scorecard- que incluye indicadores tradicionales como los económicofinancieros, ampliándolos a medidas operacionales de satisfacción de los usuarios/clientes. Además de métricas de los procesos internos y en las actividades de innovación, así como la mejora continua en la gestión. Todos estos indicadores resultan claves para visualizar los avances en la planificación estratégica efectuada y mejorar la toma de decisiones.

El concepto de "Calidad" ha venido evolucionando hacia el concepto de Calidad Total que es mucho más amplio y no está enfocado en el producto sino en la calidad de toda la organización. Para poder "tangibilizar" la Calidad Total se desarrollaron distintos modelos de Calidad Total como el de la EFQM (1991)

El objetivo del presente trabajo es:

1. Desarrollar un método integrado de Gestión Universitaria basado en el BSC y el EFQM.

2. Aplicar el Método propuesto en la Universidad Católica Santa María de Arequipa

3. Analizar las ventajas de su implementación 


\section{PLANEAMIENTO ESTRATÉGICO}

Las organizaciones basadas en la estrategia utilizan un proceso de "doble lazo" que integra la gestión de presupuestos y operaciones con la gestión de la estrategia. Según Thompson (2005), un sistema de informes basado en BSC permite monitorear la evolución frente a la estrategia y tomar las medidas correctivas necesarias

\section{Cuadro Nro. 1. La Estrategia, el Balanced Scorecard y el Presupuesto}

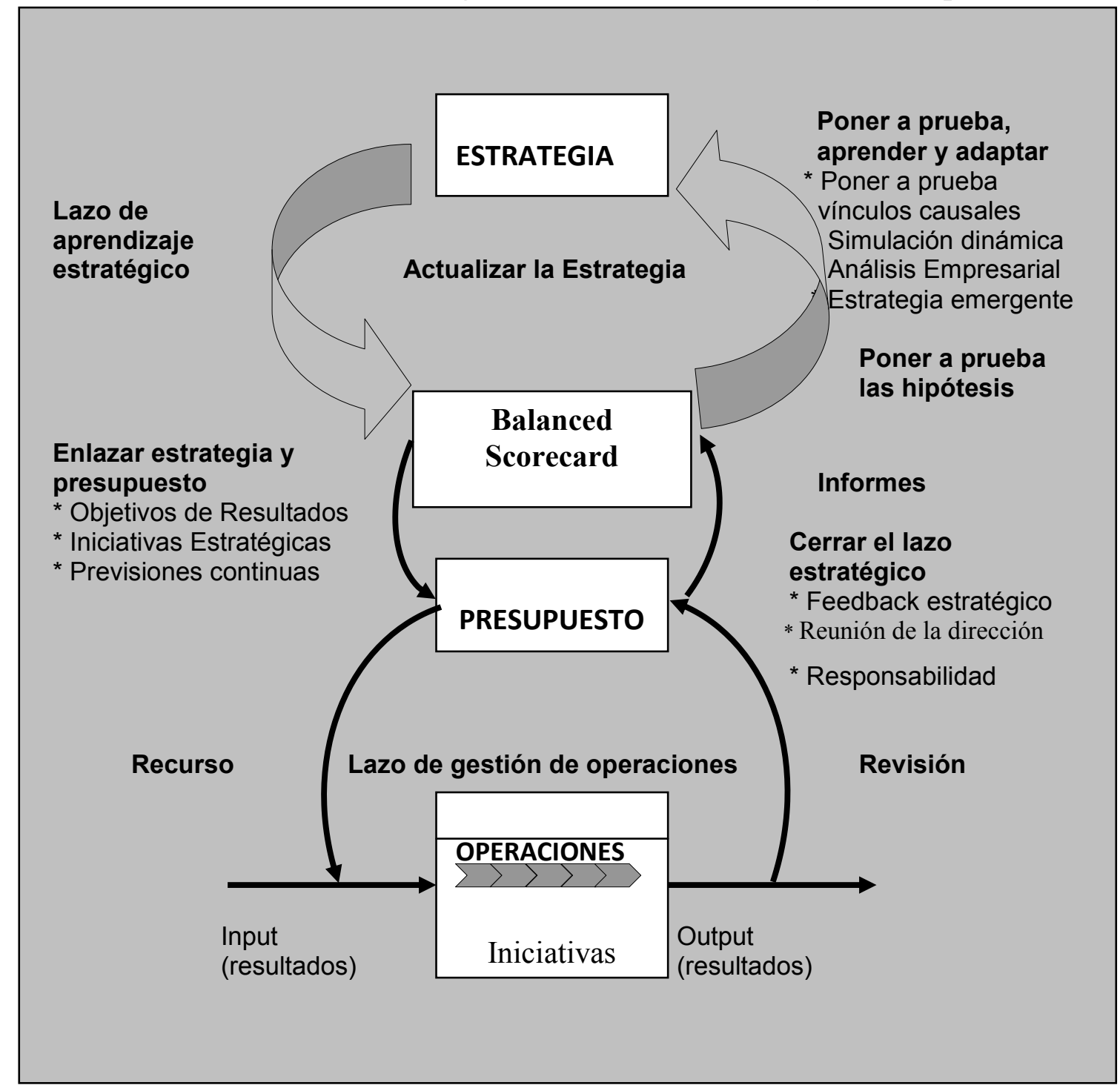

Fuente: Thompson, 2005.

La aplicación del BSC empieza con la definición de la Misión, Visión y los Valores de la organización, elementos primordiales que sólo al ser bien conceptualizados, le darán consistencia a la estrategia de la organización. Los elementos 
del Balanced Scorecard son tres: el Mapa Estratégico, la Matriz de Planificación y el Software de Control.

\section{EL MODELO EFQM}

El Modelo EFQM de Excelencia es un marco de trabajo no-prescriptivo basado en nueve criterios, que puede utilizarse para evaluar el progreso de una organización hacia la Excelencia. El Modelo establece que la excelencia sostenida en todos los resultados de una organización se puede lograr mediante distintos enfoques. (Massó, 2000)

\section{Cuadro Nro. 2. EI Modelo E.F.Q.M.}

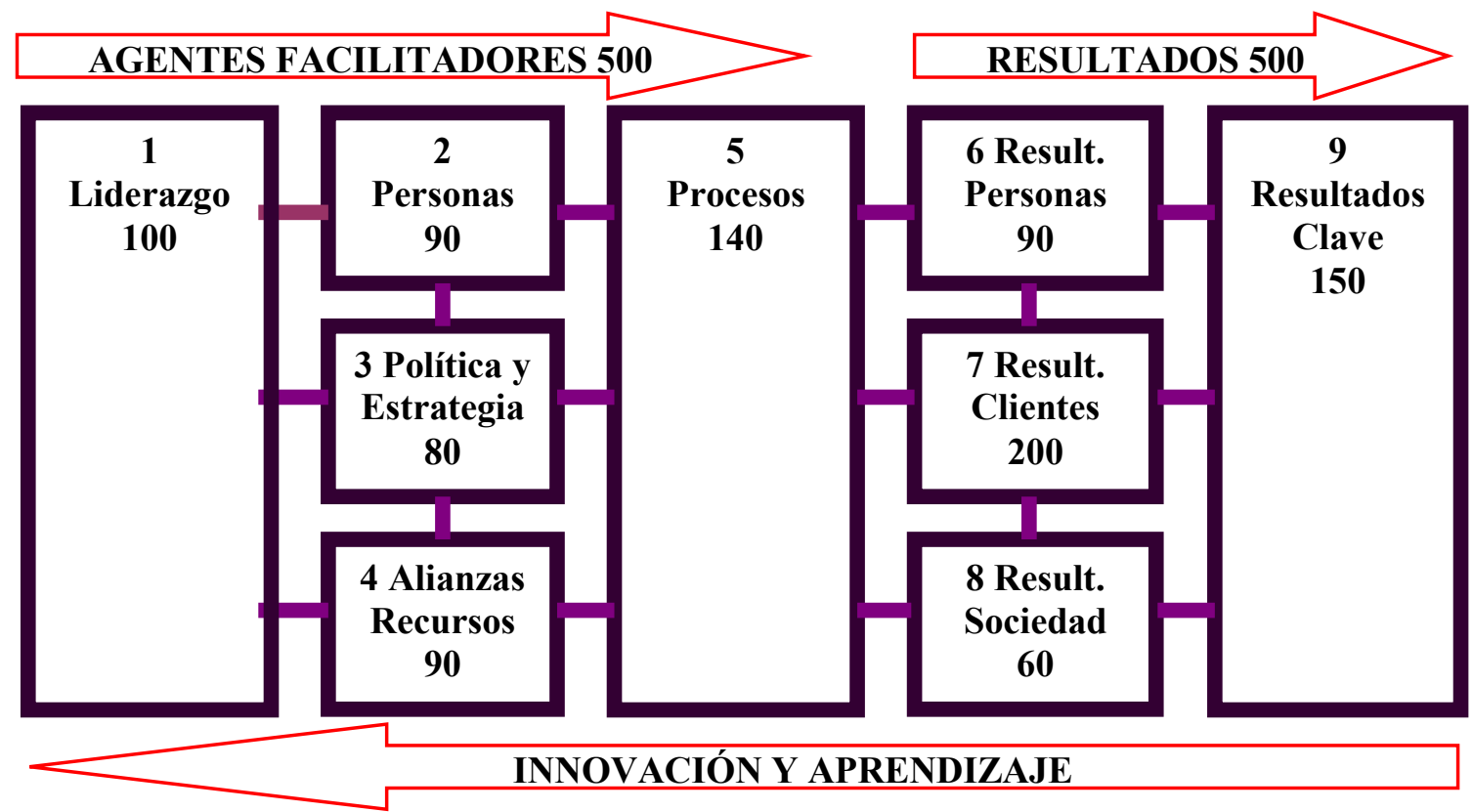

Fuente: Membrado, 1999

\section{SIMILITUDES Y DIFERENCIAS DEL EFQM Y BSC}

Para el desarrollo del modelo integrado se analizarán las similitudes y diferencias de ambas herramientas de Gestión, para buscando las fortalezas de cada uno, generar un Método Integrado

Los criterios del EFQM son 9 y las perspectivas del BSC son 4. Los aspectos comunes del BSC y el EFQM son:

Criterio 9 (Resultados Clave) y perspectiva D (financiera), Los 2 se enfocan en los resultados finales de la organización, es decir, los resultados financieros que se encuentran definidos en los resultados clave

Criterio 7 (Resultados en los Clientes) y perspectiva C (Clientes). El BSC y el EFQM consideran que el cliente es el que crea valor para la organización y que de su nivel de satisfacción va a depender los resultados de la Organización

Criterio 5 (Procesos) y perspectiva B (Procesos Internos). Ambos consideran que hay que incidir en los procesos para lograr una adecuada satisfacción del cliente que es el que al final de cuentas define la calidad 
Criterio 2 (Personas), 6 (Resultados en las Personas) y perspectiva A (Aprendizaje y Desarrollo). Los trabajadores son importantes en el caso del EFQM, para la Gestión de la Calidad y en el caso del BSC para el logro de la Visión y Misión de la Organización

Todas las perspectivas del BSC se encuentran contenidas en los criterios del EFQM, pero no todos los criterios del EFQM se encuentran explícitamente identificados en las perspectivas del BSC.

\section{Cuadro Nro. 3. Diferencias y Similitudes del BSC y el EFQM}

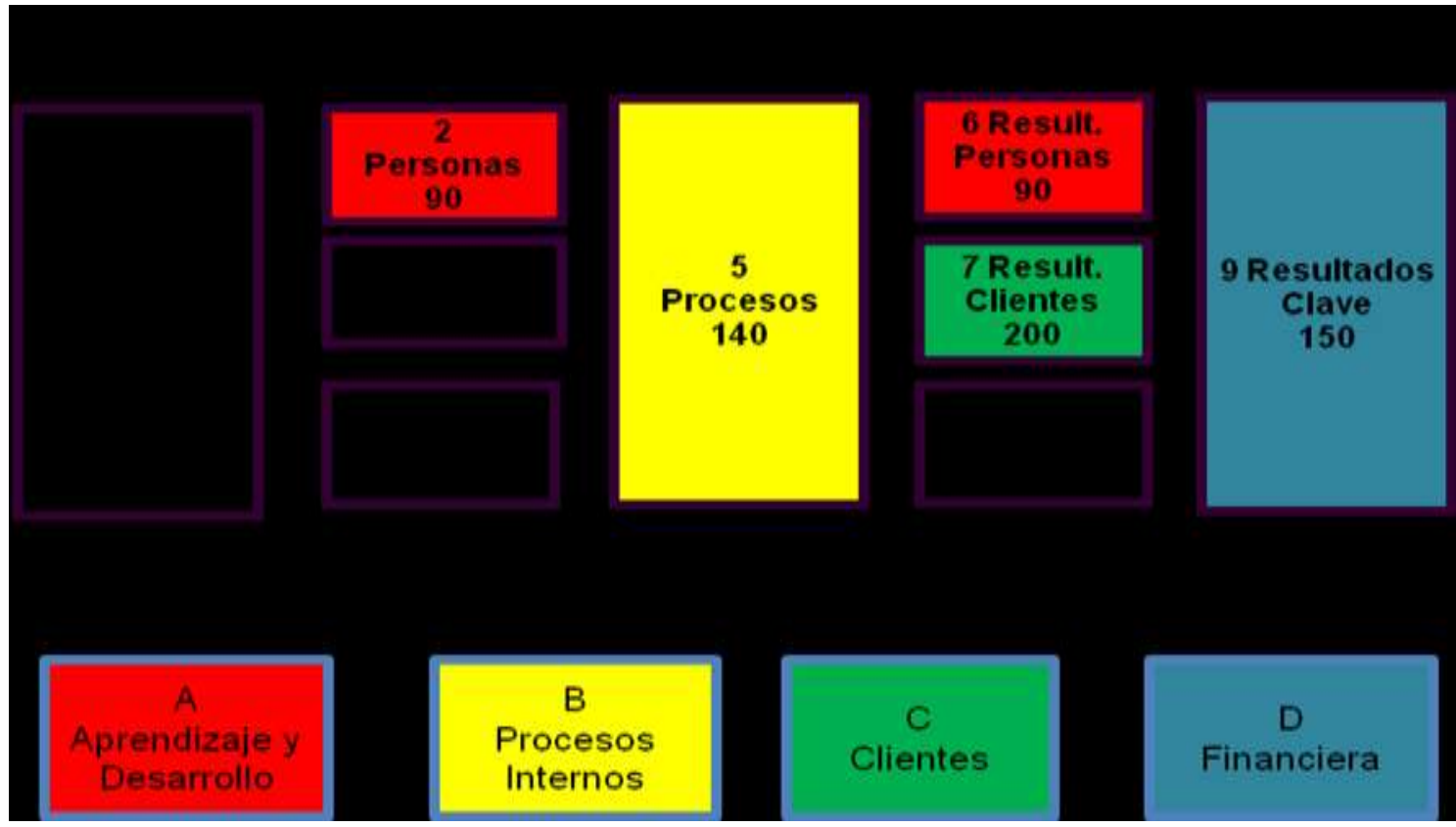

Fuente: Elaboración propia

El liderazgo, Política y Estrategia, Alianzas y Recursos y Resultados en la Sociedad, no los contempla el BSC, siendo aspectos importantes para el desarrollo sostenible de cualquier Institución, por lo que se considera desarrollar un Mapa Estratégico Genérico tomando como base los 9 criterios del EFQM

$\mathrm{Al}$ integrar el EFQM con el BSC, aumentamos las perspectivas originales del BSC en el Mapa Estratégico, enfocándolo hacia la calidad, y al aplicar la metodología del BSC, operativizamos el modelo EFQM. Es decir, que los objetivos cualitativos se miden a través de indicadores; además que se traducen en Inductores, e Iniciativas sujetas a programación y control.

Es importante recalcar que no se debe considerar un Método único, el Método debe adaptarse a las características de cada Institución Universitaria, pero debe tener como base los 9 criterios del EFQM. La adaptación a cada Institución debe tener en cuenta su Visión, Misión y Valores. El Método genérico propuesto se muestra en el Grafico Nro. 1. 


\section{APLICACIÓN DEL MÉTODO A LA UNIVERSIDAD CATÓLICA DE SANTA MARÍA}

La Universidad Católica de Santa María es una comunidad integrada por profesores, estudiantes y graduados.

\section{VISIÓN}

La Universidad Católica de Santa María, acredita niveles superiores de calidad, competitividad, ética y excelencia en el cumplimiento de su misión institucional; y contribuye a la descentralización y fortalecimiento de la Macro-Región Sur, consolidando a Arequipa, como Patrimonio Cultural de la Humanidad, bajo una concepción humanística y cristiana. (www.ucsm.edu.pe)

\section{MISIÓN}

La Universidad Católica de Santa María, está dedicada a la formación personal, académica y profesional permanente del estudiante, con una sólida base humanística, quien recibe en ejercicio de sus capacidades, la orientación continua para lograr su desarrollo integral.

Concede especial importancia a la investigación científica y tecnológica, coordinada con una sostenida acción de proyección y extensión universitaria.

Propende la vinculación e inserción permanente con las actividades productivas de la sociedad, buscando contribuir al desarrollo integral de la región y el país, bajo una concepción humanística y cristiana. (www.ucsm.edu.pe)

\section{Gráfico Nro. 1. Método Integrado Final}

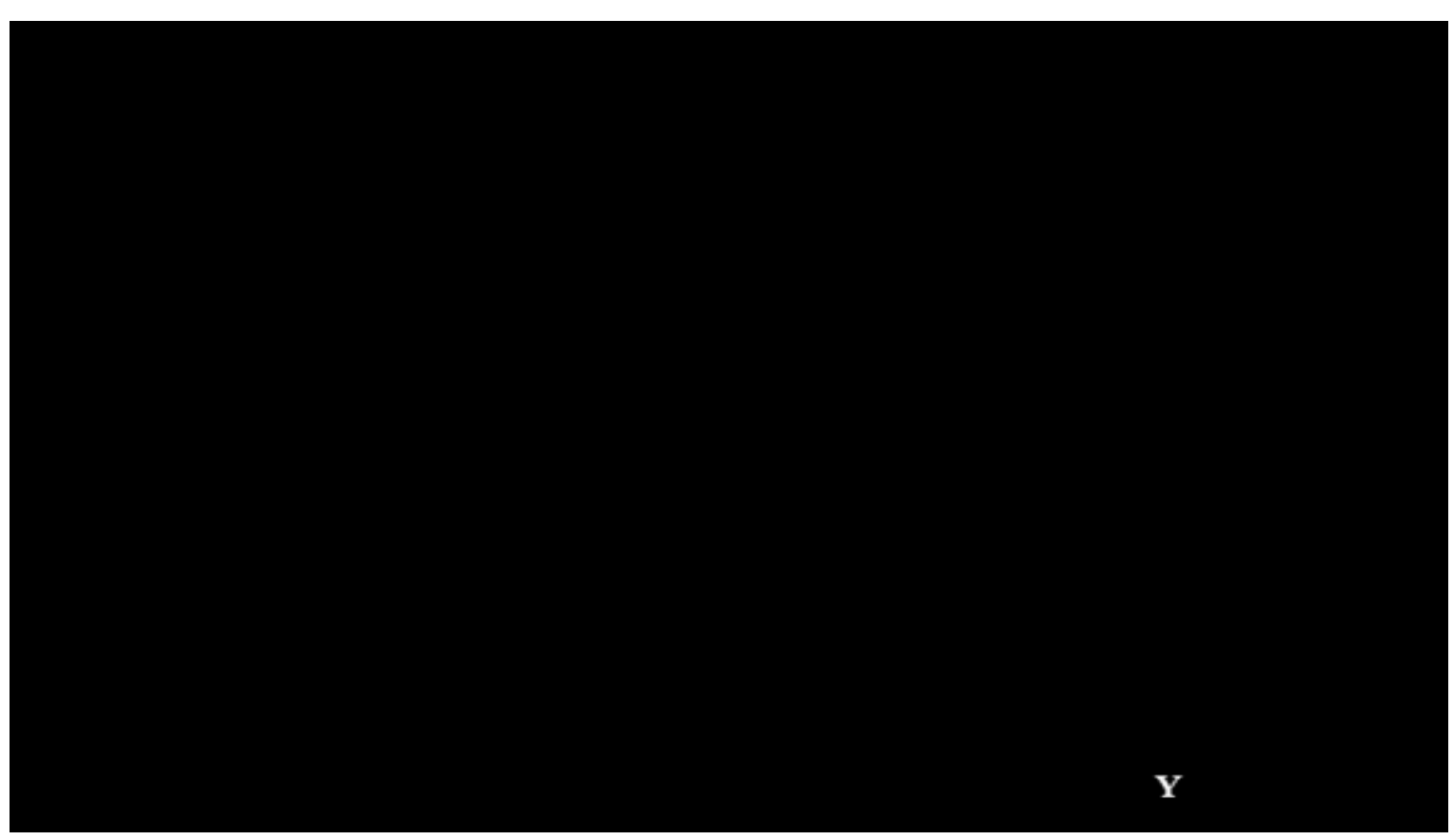

Fuente: Elaboración propia 
La Universidad Católica de Santa María como se desprende de su Visión y Misión no tiene como objetivo final resultados económicos. Pero siendo el aspecto financiero importante para su sostenibilidad es que se ha considerado un criterio adicional en la base del Modelo propuesto

\section{Grafico $N^{\circ}$ 2. Mapa Estratégico propuesto para la UCSM}

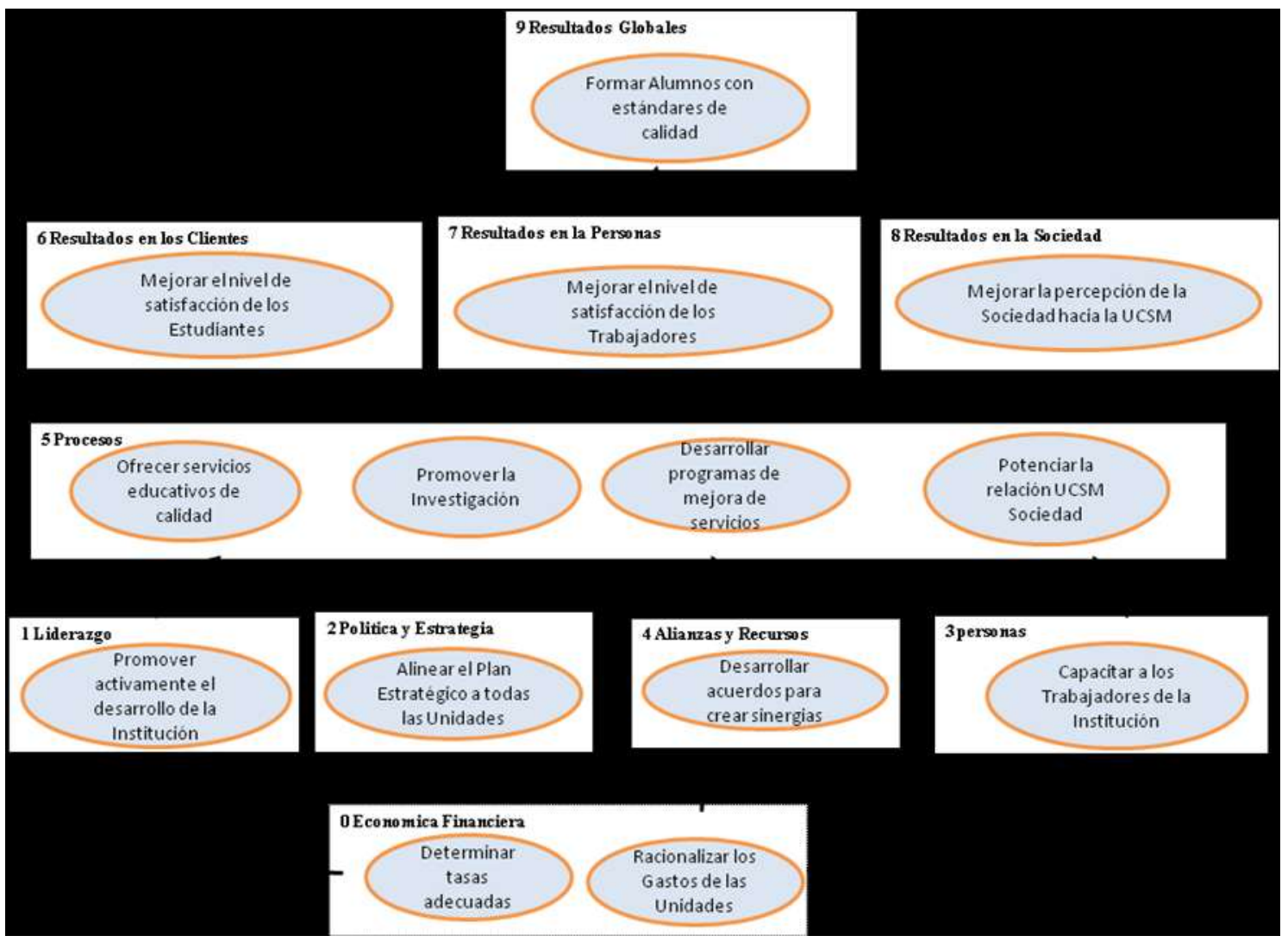

Fuente: Elaboración propia

Para cada objetivo del Mapa Estratégico, se desarrolla una matriz de Planificación, que operativiza en Proyectos y actividades el logro de dicho Objetivo. A modo de ejemplo se presenta en el Cuadro Nro. 3 la Matriz de Planificación de uno de los 14 objetivos: Determinar tasas Adecuadas, que corresponde a la Perspectiva Económica Financiera. Debe desarrollarse una Matriz de Planificación por cada Objetivo. En el caso del ejemplo existen 14 objetivos. Esta Matriz de Planificación permitirá el control del avance del logro de cada uno de los objetivos propuestos.

Del mismo modo también debe desarrollarse una descripción de cada uno de los indicadores de la Matriz de Planificación, para eliminar errores de interpretación al momento de controlar el avance de cada Objetivo.

Para el Control del cumplimiento de cada objetivo se debe aplicar la medición, que esta explicada en la descripción del indicador, para el Objetivo: Determinar Tasas Adecuadas, la descripción del Indicador Percepción del Mercado Objetivo (PMO), 
indica que debe realizarse una encuesta para determinar el Nivel de Conformidad del Mercado Objetivo con las Tasas cobradas. Un ejemplo del cálculo se muestra en el Cuadro Nro. 6.

\section{Cuadro Nro. 4. Perspectiva 0 - Económica Financiera - Determinar Tasas Adecuadas}

\begin{tabular}{|c|c|c|c|c|c|c|c|}
\hline Objetivos & Indicador & Metas & Inductor & Iniciativas & Inicio & Fin & Costo \\
\hline \multirow{7}{*}{$\begin{array}{l}\text { Determinar } \\
\text { tasas } \\
\text { adecuadas }\end{array}$} & \multirow{7}{*}{$\begin{array}{c}\% \text { de } \\
\text { Alumnos } \\
\text { conformes }\end{array}$} & \multirow{7}{*}{$\begin{array}{l}2010>60 \% \\
2011>65 \% \\
2012>70 \%\end{array}$} & \multirow[b]{2}{*}{$\begin{array}{c}1 \\
\text { Marketing }\end{array}$} & $\begin{array}{l}11 \text { Definición del } \\
\text { Público Objetivo de la } \\
\text { Universidad tanto en } \\
\text { Pre como Post Grado }\end{array}$ & 02/11/2009 & $31 / 12 / 2009$ & 200 \\
\hline & & & & $\begin{array}{l}12 \text { Determinación de } \\
\text { los costos reales } x \\
\text { Programa Profesional } \\
\text { en el Pre y Post } \\
\text { Grado } \\
\end{array}$ & 01/10/2009 & $31 / 12 / 2009$ & 200 \\
\hline & & & \multirow{5}{*}{$\begin{array}{c}2 \\
\text { Contabilidad }\end{array}$} & $\begin{array}{l}21 \text { Determinación de } \\
\text { los Márgenes de } \\
\text { Utilidad adecuados en } \\
\text { cada Programa }\end{array}$ & 02/01/2010 & $31 / 01 / 2010$ & 400 \\
\hline & & & & $\begin{array}{l}22 \text { Definir los Costos } \\
\text { Reales de los } \\
\text { diferentes Servicios: } \\
\text { Cursos x Jurado, } \\
\text { Bachillerato, } \\
\text { Titulación, etc. } \\
\end{array}$ & 02/01/2010 & $31 / 01 / 2010$ & 1,500 \\
\hline & & & & $\begin{array}{l}23 \text { Determinación de } \\
\text { los Índices de } \\
\text { Incremento Anual de } \\
\text { Servicios }\end{array}$ & 01/03/2010 & $31 / 03 / 2010$ & 300 \\
\hline & & & & $\begin{array}{l}24 \text { Determinación de } \\
\text { las diferentes } \\
\text { Categorías de Tasas }\end{array}$ & 01/03/2010 & $30 / 03 / 2010$ & 200 \\
\hline & & & & $\begin{array}{l}25 \text { Análisis de la } \\
\text { Competencia de la } \\
\text { Universidad x } \\
\text { Programa }\end{array}$ & $01 / 12 / 2009$ & $31 / 01 / 2010$ & 2,000 \\
\hline
\end{tabular}

Fuente: Elaboración propia 
Cuadro Nro. 5. Ejemplo del Descripción del Indicador Percepción del Mercado Objetivo (PMO) para el Software del BSC

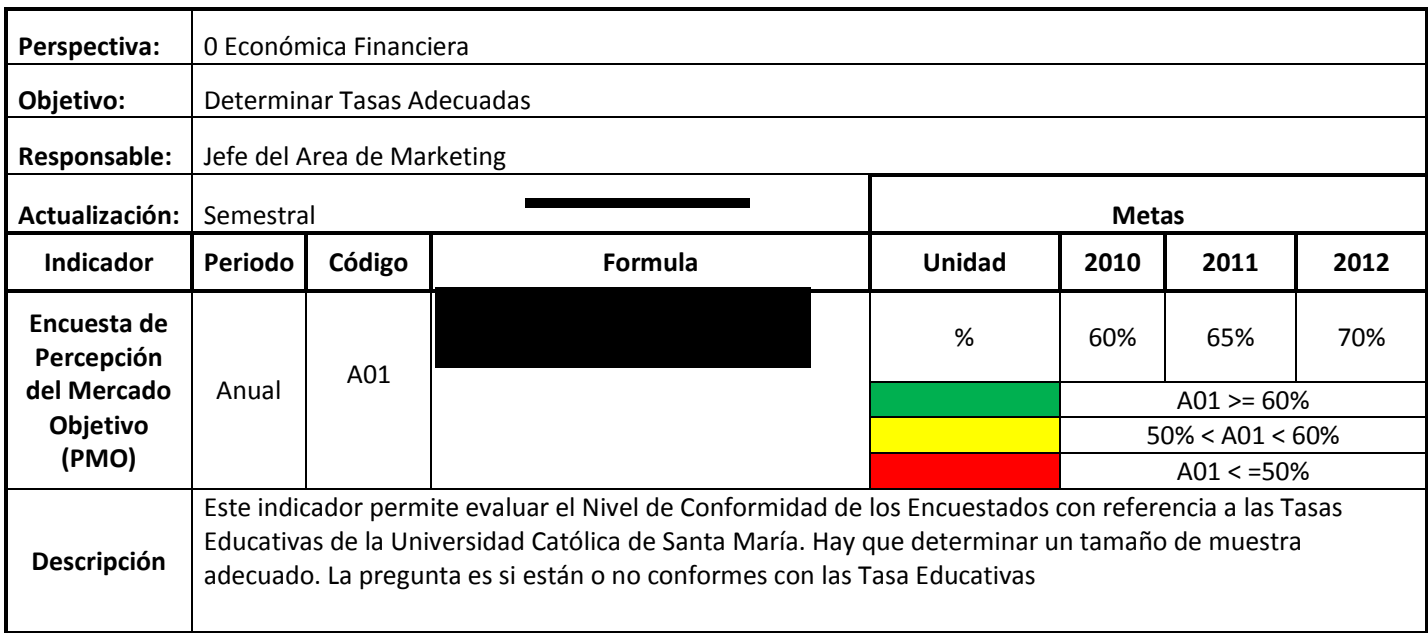

Fuente: Elaboración propia

Cuadro Nro. 6. Ejemplo de Cálculo de Indicador Encuesta de Percepción del Mercado Objetivo (PMO

\begin{tabular}{|l|r|r|r|r|}
\hline & & 2010 & 2011 & 2012 \\
\hline Completamente de Acuerdo & 5 & 60 & & \\
\hline Parcialmente de Acuerdo & 4 & 0 & & \\
\hline De acuerdo & 3 & 135 & & \\
\hline Parcialmente en Desacuerdo & 2 & 125 & & \\
\hline Totalmente en desacuerdo & 1 & 45 & & \\
\hline & & 365 & & \\
\cline { 1 - 4 } & Indicador & $\mathbf{5 3 \%}$ & & \\
\cline { 2 - 4 } & &
\end{tabular}

Fuente: Elaboración propia

\section{CARACTERÍSTICAS DEL MÉTODO PROPUESTO}

El Método Integrado BSC - EFQM, presenta las siguientes características:

Desarrolla un Mapa Estratégico para la U.C.S.M. Este Mapa Estratégico, el mismo que se basa en un análisis causa - efecto del BSC, se realiza en función de la Misión y Visión de la Universidad, lo que nos muestra una jerarquía de metas. Esta basado en los 9 Criterios del EFQM, habiéndose desarrollado objetivos para cada uno de estos 9 Criterios. El criterio 5 Procesos, ha sido dividido en 4 objetivos específicos

Desarrolla una Matriz de Planificación. A partir del Mapa Estratégico se elabora para cada uno de los 14 objetivos del Mapa, una matriz de Planificación, donde se indican: los inductores, sobre los que hay que actuar, las metas que se deben lograr en los próximos 3 años, las iniciativas que hay que desarrollar para el logro de objetivo propuesto, el inicio y fin de cada iniciativa, así como el costo estimado, el Responsable 
de cada iniciativa, así como la forma en que se debe reportar el avance y la frecuencia del mismo.

Es importante la asignación de responsables del Método ya que permitirá analizar el nivel de contribución de los miembros de la Organización al logro de metas y podrá desarrollarse una Administración por Objetivos.

Desarrolla un Software de Control. Este software para este caso ha sido desarrollado en Excel y debería estar relacionado con el Software de la U.C.S.M., lo que le permitiría estar actualizado permanentemente.

Debe lograrse la participación de todas las áreas en su elaboración. En el desarrollo del mapa estratégico como de la Matriz de Planificación debe lograrse la participación de la mayor cantidad de trabajadores de la Institución. Ello permitirá, enriquecer su desarrollo con los aportes de los trabajadores y lograr que los trabajadores sientan como "suyo" el Plan.

\section{VENTAJAS DEL MÉTODO PROPUESTO}

Permite desarrollar una Autoevaluación de la Institución. El Método al estar basado en el Modelo EFQM, permitirá que la U.C.S.M. realice una autoevaluación permanente de su nivel de excelencia, ya que el EFQM mide el Nivel de Excelencia de la Institución sobre un puntaje máximo de 1000 puntos como lo muestra el Cuadro Nro. 2. Ello permitirá medir el nivel de Excelencia de la U.C.S.M.

Facilita la Implementación de un Presupuesto en función de Objetivos. La asignación del presupuesto debería hacerse en función de los requerimientos, cada unidad en función de las metas planteadas debería demandar dinero para la ejecución del mismo, el que ya se encuentra especificado en la columna Costo de cada una de las iniciativas.

Este presupuesto para cada año debería ser reformulado en función del cumplimiento o no de los objetivos planteados, de modo que permitiría premiar presupuestalmente a las Unidades más eficientes.

Permite una Gestión Estratégica efectiva y eficiente de la Universidad. El método integrado facilita la formulación, implementación y control de un Plan Estratégico para la Universidad, en el corto y mediano plazo.

\section{FASES DE LA IMPLEMENTACIÓN DEL MÉTODO}

Para la Implementación del Método Propuesto se ha considerado que deben desarrollarse las siguientes fases:

1. Decidir políticamente la Implementación del Método. Es necesario para la Implementación que los más Altos Directivos de la Institución estén convencidos de su implementación, para poder alentar a todos los niveles de la Organización

2. Constituir una Comisión de Implementación. En la misma deberán sentirse representados todas las áreas de la Institución, de esta forma se logrará que la mayoría de trabajadores se sienta parte del Método a implementar y no sientan que es un procedimiento impuesto de la Dirección.

3. Desarrollar una Autoevaluación de la Institución. Realizar una autoevaluación para determinar en que nivel de Excelencia en cada una de las 10 perspectivas planteadas se encuentra la Institución

4. Determinación de las perspectivas requeridas. En el caso de la Universidad Católica de Santa María se han considerado 10 perspectivas, los 9 criterios 
del EFQM, a los cuales se le ha adicionado en la base del Modelo una Perspectiva Financiera, dado que la Universidad no tiene fines de lucro. Dependiendo de los Factores Claves de Suceso de la Institución puede reformularse dichos criterios, tanto en cantidad, como en el Orden de los mismos, para poder alinearlos a su Visión y Misión.

5. Desarrollar el Mapa Estratégico. En función de las Perspectivas definidas en el punto anterior hay que desarrollar un Mapa Estratégico, para lo cual se deben plantear uno o varios objetivos por cada una las perspectivas, se puede tomar como ejemplo el Gráfico Nro. 2.

6. Desarrollar la Matriz de Planificación. Por cada objetivo propuesto se debe indicar las iniciativas que nos van a permitir el logro de dicho objetivo, los inductores, los responsables, la fecha de inicio y culminación de cada iniciativa. Así como el indicador que nos permitirá controlar el logro de nuestro objetivo, se puede tomar como ejemplo el Cuadro Nro. 4.

7. Detallar la especificación del indicador. Para cada indicador detallar claramente la formula como se va a calcular dicho indicador, también determinar los rangos entre los cuales el indicador es satisfactorio (verde), alerta (amarillo) o insatisfactorio (rojo), así como lo muestra el Cuadro Nro. 5.

8. Desarrollar el Software de Control. Es importante evaluar permanentemente a la Organización a través de los indicadores, para ello es necesario desarrollar un software que nos indique en que nivel de avance se encuentra cada indicador, lo ideal es que dicha información sea alimentada en línea con los Sistemas de Información.

9. Controlar el Modelo a través del Software de Control. Analizar si la Organización esta operando de acuerdo a lo Planificado, para tomar medidas correctivas o retroalimentar el Método, y actualizarlo en cualquiera de las fases antes descritas.

\section{Conclusiones}

No existe un Modelo de Calidad, Herramienta de Gestión ni Método que solucione la complejidad de la Gestión de las Universidades. El Método propuesto integra el Balanced Scorecard, que es una buena herramienta de Gestión y el EFQM que es uno de los Modelos de Calidad más utilizados con mayor éxito a nivel mundial. Mediante el Método Integrado BSC EFQM propuesto se la demostrado que es factible la implementación de un Método de gestión de las Universidades basado en una herramienta de Planificación Estratégica: el Balanced Scorecard y un Modelo de Gestión de Calidad: el Modelo Europeo de Calidad EFQM

Para el desarrollo del Método Integrado propuesto, se ha analizado la interrelación existente entre el BSC y el EFQM, mientras el primero se enfoca en el logro de la Visión, el segundo hace énfasis en la Calidad para el logro de la excelencia, pero ambos tiene como base la satisfacción de los clientes y el logro de los objetivos de la Institución. La forma como se interrelacionan se muestra en el Cuadro Nro. 3.

El método Propuesto consta de un Mapa Estratégico de 10 Perspectivas (Gráfico Nro. 2.), una Matriz de Planificación, cuyo ejemplo se muestra en el Cuadro Nro. 4.

Se ha evaluado la el Método Integrado BSC EFQM Propuesto en la U.C.S.M., analizándose las ventajas, características y etapas de la implementación. 


\section{BIBLIOGRAFIA}

European Foundation for Quality Management. Pagina Oficial del Modelo Europeo de Calidad EFQM. Guía en español. Disponible en

$<$ http://www.efqm.org/uploads/introducing_spanish.pdf. 2008>

FOSCA, Carlos; Wanuz, Karina; Cueva Ricardo. Gestión De La Calidad Universitaria: El Caso De La P. U. C. P. Dirección Académica de Planeamiento y Evaluación Pontificia Universidad Católica del Perú. 2003.

KAPLAN, Robert; NORTON, David. EI Cuadro de Mando Integral: (The Balanced Scorecard). España: Editorial Gestión 2000 S. A. 1997.

KAPLAN, Robert; NORTON, David. Como utilizar el Cuadro de Mando Integral Para Implementar y gestionar su estrategia. España: Harvard Business School Publishing Corporation, España. 2001.

MASSÓ PEREZ, Xavier; TORT-MARTORELL LLABRÉS, Xavier. El modelo EFQM Aplicado a la Universidad. Un caso práctico. España: Editorial UPC. 2000.

MEMBRADO Martínez, Joaquín. La Gestión Empresarial A Través Del Modelo Europeo De Excelencia De La E.F.Q.M. España: Diaz de Santos S.A. 1999

Pontificia Universidad Católica del Perú. PUCP Plan Estratégico 2007 - 2010. Perú: Editorial PUCP. 2007.

THOMPSON, Artur; STRICKLAND, Alonzo. Administración Estratégica. Conceptos y Casos. Mexico: Mc Graw Hill 2005.

Universidad Católica de Santa María. Misión y Visión de la UCSM. Disponible en $<$ http://www.ucsm.edu.pe>.

Universidad de Alicante. Marco para el Plan Estratégico de la Universidad de Alicante (Horizonte 2012) Planificación Estratégica Universidad de Alicante. Disponible en <http://www.ua.es>.

Universidad Miguel Hernández. Los indicadores y la Gestión de las Unidades. I Jornada de Formación. La dirección estratégica en el ámbito universitario Plan Estratégico de la Universidad Miguel Hernández. Disponible en

$<$ http://www.umh.es $>$. 